\title{
Recommendation of use of checklists in tibial intramedullary nail removal: Retrospective study of mechanical complications related to nail removal
}

\author{
Antti Stenroos*, Tuomas Brinck, Lauri Handolin \\ Department of Orthopedics and Traumatology, University of Helsinki and Helsinki, University Hospital, PO Box 266, FI-00026 HUS Finland
}

\section{A R T I C L E I N F O}

\section{Article history:}

Accepted 9 May 2018

\section{Keywords:}

Tibia fracture

Iatrogenic fracture

Intramedullary nail removal

Complication

\begin{abstract}
A B S T R A C T
Background: The removal of implants such as intramedullary nails is one of the most common operations in orthopedic surgery. The indications for orthopedic implants removal will always remain a subject of conversation and hardly supported by literature. The aim of this study to report injuries of treatment in tibial nail removal and to determine if there are fracture characteristics, patient demographics, or surgical details that may predict a complication.

Methods: This is a retrospective seven-year (2010-2016) study including a total of 389 tibial intramedullary nail removals at the Helsinki University Hospital's orthopedic unit. Patients with tibial fracture and removal of intramedullary nail were identified from the hospital discharge register and analyzed.

Results: A total of $21(5,4 \%)$ nail removal related mechanical complications (iatrogenic fractures, nerve injuries, failures to remove the nail) were noted. The most common complication was iatrogenic fracture $(\mathrm{n}=15,3,8 \%)$. In $6 / 15$ cases the fracture was caused by broken interlocking screws, In 5/15 cases the iatrogenic fracture was caused accidentally by extracting the nail without prior removal of all distal interlocking screws. In one case, new condensed bone had formed around the nail's distal end and case the forced nail extraction caused a re-fracture in both tibia and fibula.

Conclusion: Nail removal can be a challenging operation which does not always receive the necessary preoperative planning or operative expertise. Iatrogenic fractures were most often caused by inadequate preoperative planning or assuming that a broken interlocking screw tilts during the extraction. We suggest the use of checklists in preoperative planning to avoid fractures caused by broken or undetected interlocking screws.
\end{abstract}

(c) 2018 Elsevier Ltd. All rights reserved.

\section{Background}

Tibial fracture is the most common long bone fracture, seen by trauma units on almost a daily basis [1], and intramedullary nails have become widely accepted as the treatment of choice [2-5].

The removal of implants such as intramedullary nails is one of the most common operations in orthopedic surgery [6]. However, despite the prevalence of the procedure, there is currently no consensus among orthopedic surgeons regarding the criteria for tibial intramedullary nail removal. The decision to remove a tibial intramedullary nail has largely been considered routine - or elected by the patient. Removal of an intramedullary nail is generally regarded as a minor, low-risk procedure with little

\footnotetext{
* Corresponding author.

E-mail address: antti.stenroos@helsinki.fi (A. Stenroos).
}

morbidity [7], even though implant removal is associated with various known complications such as re-fracture, hematoma, lengthy operating times and implant breakage [7-13].

Within the literature, previously listed criteria for implant removal included: symptomatic hardware, skeletally immature patients, broken hardware, compromised skin, nonunion, malunion, infection, fear of carcinogenesis, peri-implant failure, prevention of postunion stress-shielding, prevention of future bacterial colonization, avoidance of difficult surgery in case of refracture or implant failure, avoidance of problems with a future joint replacement, and the possibility that removal will improve functional outcome $[10,11,14-16]$.

The aim of this study is two-fold: 1) to report the unplanned events and injuries of treatment of a large retrospective series of patients who underwent a tibial nail removal operation at a single academic institution (Helsinki University Hospital's orthopedic trauma unit); and 2) to determine if there are fracture 
characteristics, patient demographics, or surgical details that may predict a complication.

\section{Materials and methods}

This is a retrospective seven-year (2010-2016) study including a total of 389 tibial intramedullary nail removals at the Helsinki University Hospital's orthopedic unit. Patients with tibial diaphyseal fracture or distal tibial fracture (ICD-10 diagnosis codes S82.2 and S82.3) and removal of intramedullary nail from lower leg (code NGU20 in NOMESCO Classification of Surgical Procedures) were identified from the hospital discharge register. Stress or pathological fractures were excluded from the study, however osteoporotic fractures were included. Also, patients who experienced nail removal during re-do nailing due to malalignment after the primary operation during the same hospital period were excluded from the study. Bilateral fractures were recorded as separate fractures. Intramedullary nails (IMN) used in our institution are titanium locked reamed tibia nails (either DePuy Synthes ETN ${ }^{\mathbb{R}}$ or Stryker $\mathrm{T} 2{ }^{\circledR}$ nails).

Hospital records were retrospectively reviewed to collect the following data: age, gender, comorbidities, patient's body mass index (BMI), documented reason for implant removal, length and diameter of the intramedullary nail, time of removal, experience of the surgeon (consultant, senior orthopedic registrar or surgical registrar). Tibia fractures were classified according to the Müller's Arbeitsgemeinschaft für Osteosynthesefragen (AO) classification with the aim of finding the fracture patterns that might be associated with complications.

Results are presented as medians and means \pm standard deviation (SD) for continuous non-skewed variables. The frequency distribution of the categorical variables is compared between the groups with the Chi-square test. The statistically significant level is set as $\mathrm{p}<0.05$. Binary regression analysis was performed to determine which parameter (age, BMI, fracture type, nail size, operator's experience) was independently of the significance for prediction of complication. Statistical program SPSS 22 (IBM Corp. released 2009. IBM SPSS Statistics for Windows, version 13.0. Armonk, NY: IBM Corp.) was used for analyzes.

\section{Results}

Overall, 389 tibial intramedullary nails $\left(\operatorname{ETN}^{\circledR}=357\right.$, Stryker $\mathrm{T} 2{ }^{\circledR}=32$ ) were removed from 385 patients at our institution over a 7-year period from January 2010 to December 2016. Of the 389 removed tibial intramedullary nails, 28 were inserted in other hospitals. During the same time period 950 patients were treated with intramedullary nails at our institution, resulting in an average $38 \%$ computational nail removal rate.

The mean IMN length was $360 \mathrm{~mm}$ (range 300-395) and mean IMN diameter was $10 \mathrm{~mm}$ (range $8-11 \mathrm{~mm}$ ). Prior the nail removal all patients received prophylactic intravenous antibiotics in operating room. Mean age of the patients was 40 years (range 23-58 years), and there was a male dominance in the study population $(n=212,55 \%)$. The mean time from IMN insertion to removal was 21 months (range 12-132 months). The mean body mass index of the patients was 26 (range 20-34). The most
Table 1

Reason for intramedullary nail removal.

\begin{tabular}{lll}
\hline & Removals & Percentage \\
\hline Routine removal & 216 & $55,5 \%$ \\
Anterior knee pain & 75 & $19,2 \%$ \\
Pain at locking screw site & 61 & $15,6 \%$ \\
Patient request & 28 & $7,2 \%$ \\
Not stated & 5 & $1,2 \%$ \\
Delayed union & 1 & $0,3 \%$ \\
Deep infection & 1 & $0,3 \%$ \\
Broken implant & 1 & $0,3 \%$ \\
Nail migration & 1 & $0,3 \%$ \\
Total & 389 & $100 \%$ \\
\hline
\end{tabular}

common reason for nail removal was routine removal $(n=216$, $55 \%)$ followed by anterior knee pain $(n=75,19 \%)$ and pain at locking screw site $(\mathrm{n}=61,16 \%)$ (Table 1$)$.

Seventy-five procedures (19\%) were performed by surgical registrars, 127 (33\%) by senior orthopedic registrars and 187 (48\%) by orthopedic consultants.

A total of $21(5,4 \%)$ nail removal related mechanical complications (iatrogenic fractures, nerve injuries, failures to remove the nail) were noted in 19 patients $(4,8 \%)$. The most common complication was iatrogenic fracture $(n=15,3,8 \%)$ More detailed information on all complications is presented on Table 2.

There were $31(8,0 \%)$ cases with broken distal interlocking screw(s). In 25/31 cases the broken screws were identified on preoperative $x$-rays and 4/31 were identified while removing the interlocking screws. There were two cases where surgeon didn't notice broken interlocking screws, but intraoperative fluoroscopy, applied after the nail failed to come out, revealed the case. In 22 cases, all parts of broken screws were removed prior the nail retraction, resulting in 9 cases where the distal part of broken screw was left in place assuming it tilts and gives away during nail extraction. In 3/9 cases the broken distal interlocking screw part tilted and caused no further harm. On the other hand, in 6/9 cases the broken left behind interlocking screw part did not tilt and caused iatrogenic fracture.

In 5 cases the iatrogenic fracture was caused accidentally by extracting the nail without prior removal of all distal interlocking screws due to misjudgment in preoperative planning. In 3/5 cases the undetected interlocking screw was accidentally left in the nail's distal oblique locking hole (Fig. 1.). In one case, a distal AP interlocking screw was partially removed and left to prevent nail rotation while attaching the nail removal instrument, but then forgotten and not removed completely prior to nail extraction. This resulted in a longitudinal anterior cortex fracture (Fig. 2). In one case, the surgeon was not able to find the distal AP interlocking screw and moved to proximal screws, forgetting then to remove the remaining distal interlocking screw and causing a complex fracture (Fig. 2.). In all 5/5 cases there were additional metal implants in distal fibula or distal tibia.

There were four iatrogenic fractures in cases where all the locking screws were removed prior the nail extraction. In one case, new condensed bone had formed around the nail's distal end (Fig. 3.) resulting in a firm consolidation. In this case the forced nail extraction caused a re-fracture in both tibia and fibula. In one case

Table 2

Detailed information on nail removal related mechanical complications in 389 operations.

\begin{tabular}{|c|c|c|c|c|}
\hline Surgeons experience & Number of removals & Iatrogenic fracture & Failure to remove & Nerve injury \\
\hline Registrar & $75(19,3 \%)$ & $4(5,3 \%)$ & $1(1,3 \%)$ & 0 \\
\hline Senior registrar & $127(32,6 \%)$ & $5(3,9 \%)$ & 0 & $2(1,6 \%)$ \\
\hline Consultant & $187(48,1 \%)$ & $6(3,2 \%)$ & $2(1,1 \%)$ & $1(0,5 \%)$ \\
\hline Total & 389 & $15(3,8 \%)$ & $3(0,7 \%)$ & $3(0,8 \%)$ \\
\hline
\end{tabular}




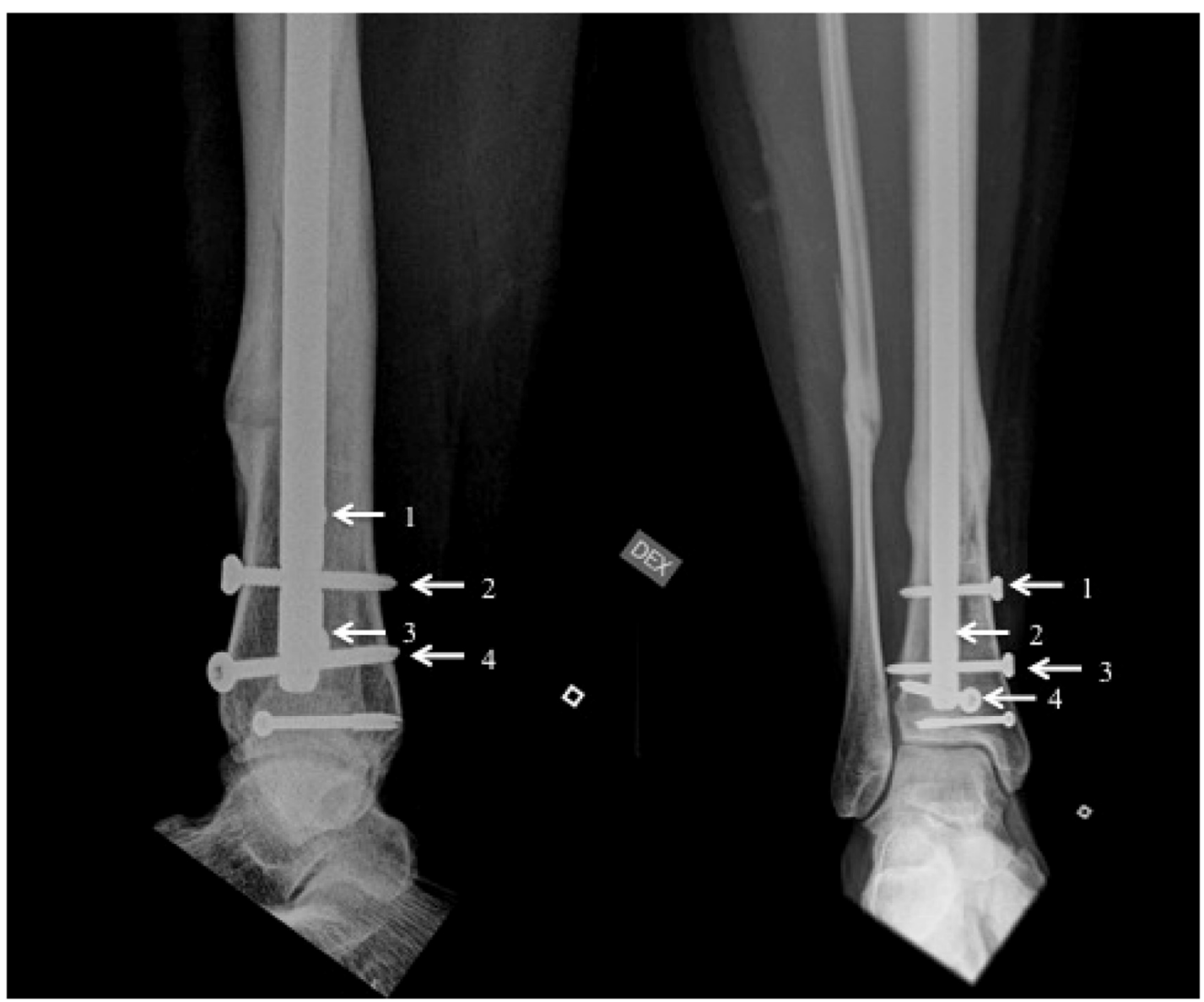

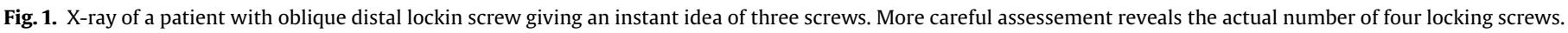

posterior tibial plateau fissure was noted perioperatively after the nail was removed and in one case a bone block broke from anterior tibial plateau. One tibial medial condyle fracture was observed four weeks after nail removal (Fig. 2). More detailed information on iatrogenic fractures is presented in Table 3.

Two patients had neuralgic pain at incision site after nail extraction (one had also an iatrogenic fracture). One patient with a complex tibia fracture (Fig. 2) suffered both sensory and motoric peroneal nerve injuries.

There were three failed attempts to remove a nail. In one case, the appropriate instrumentation (Targon ${ }^{\mathbb{R}} \mathrm{T}$ universal interlocking nail) was not available for the nail that had been implanted in another hospital. In two cases the nail was buried so deep that removal would have caused unacceptable destruction of the tibia plateau and the surgeon decided to abandon the operation.

None of the surgeons caused more than one iatrogenic fracture. There were no statistically significant differences in iatrogenic fracture rate between surgical registrars, senior orthopedic registrars or consultants ( $5 \%$ vs. $4 \%$ vs. $3 \%, p=0.72$ ). Our attempt to establish a pattern of injury and fracture type among the patients who suffered a mechanical complication failed. All complications were associated with $\mathrm{ETN}^{\circledR}$, however, due to the small sample size of $\mathrm{T}^{\circledR}{ }^{\circledR}$ we could not find any statistical significant differences between $\mathrm{ETN}^{\circledR}$ and $\mathrm{T} 2{ }^{\circledR}$. Age, sex, BMI, AO fracture type, time to nail removal, operator's experience, nail size, presence of infection or other illnesses showed no statistically significant correlation with complications.

\section{Discussion}

The iatrogenic fracture rate is high in our institution. Iatrogenic fractures were most often caused by inadequate preoperative planning or assuming that a broken interlocking screw tilts during the extraction.

In earlier studies on IMN removal, re-fracture and iatrogenic fracture rate has been generally low (0-3\%) [8,10-13,17-22]. Most of the fractures caused by nail extraction are limited to case reports [21,23-26], Sanderson [27], Boerger [7] and Hora [12] each reported one fracture and White [13] reported 2 cases. We believe that fractures caused by nail extraction are an unfortunate and serious complication that is an under-reported in literature even though the only available prospective data (Vos et al. [17]) found no re-fractures or iatrogenic fractures. Though, most INM removal studies investigate the indications and relief of symptoms after removal instead of focusing on the complications. Folwacnzny et al. [9] reported 6/103 iatrogenic fracture rate, which is relative similar to our results. Though, in their study all patients had $A C E^{\circledR}$-tibia nail with a distal $5^{\circ}$ bend towards the ventral, which has been associated with iatrogenic fractures in several case reports [2426].

Retrospectively, more than half of these iatrogenic fractures in our study could have been avoided by adequate preoperative planning and by continuous internal institutional assessment of treatment quality, which should be a part of modern fracture treatment. Causing an iatrogenic fracture is a lesson a surgeon can 


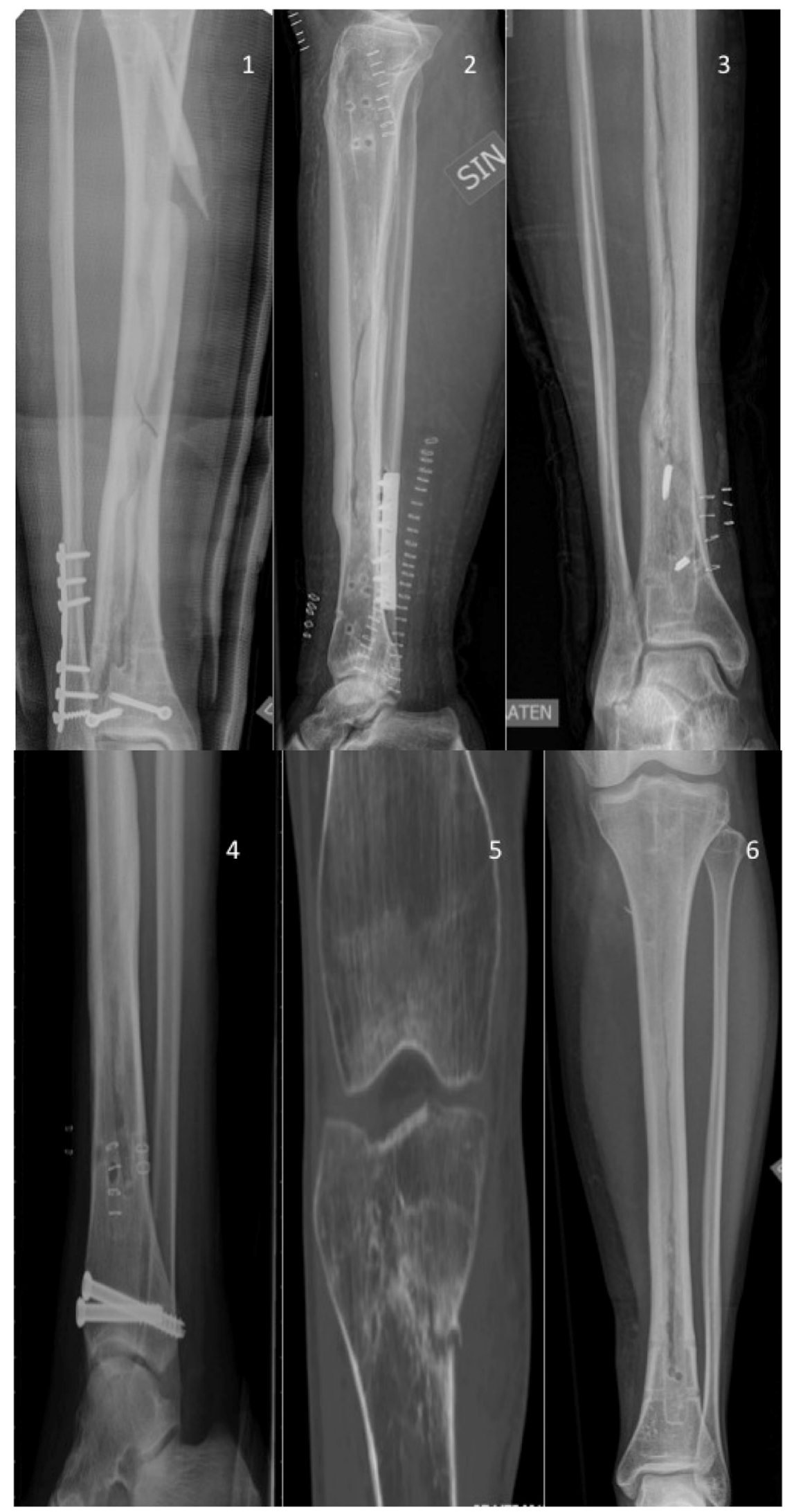

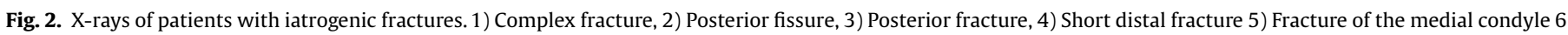
Anterior fracture. 


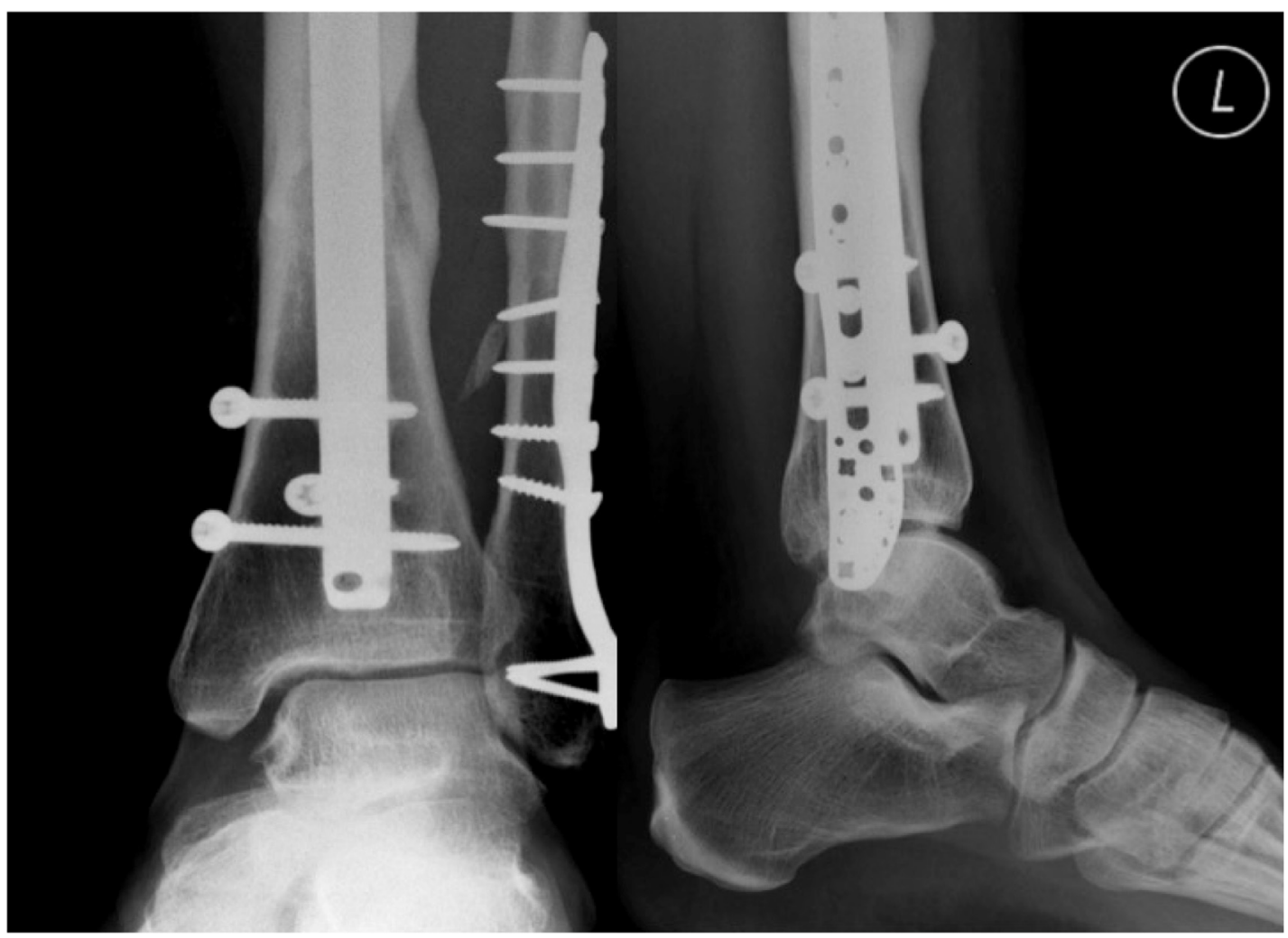

Fig. 3. New condesed bone formation through the intramedullary empty screw hole.

Table 3

Types and treatment of 15 nail removal related iatrogenic fractures in 389 operations.

\begin{tabular}{|c|c|c|c|}
\hline Cause of fracture & Surgeons experience & Type of fracture & Treatment \\
\hline Broken screw not tilting & Registrar & Longitudinal fissure & Long leg cast 4 weeks \\
\hline Broken screw not tilting & Registrar & Longitudinal fracture & Long leg cast 6 weeks \\
\hline Broken screw not tilting & Senior registrar & Complex mid diaphysis & Re-nailing \\
\hline Broken screw not tilting & Consultant & Short distal fissure & NWB* 6 weeks \\
\hline Broken screw not tilting & Registrar & Short distal fissure & NWB 6 weeks \\
\hline Broken screw not tilting & Senior registrar & Longitudinal fissure & Long leg cast 4 weeks \\
\hline Undetected oblique screw & Registrar & $10 \mathrm{~cm}$ distal groove & Long leg cast 6 weeks \\
\hline Undetected oblique screw & Consultant & Longitudinal fracture & Long leg cast 12 weeks \\
\hline Undetected oblique screw & Consultant & Longitudinal fracture & Long leg cast 12 weeks \\
\hline Forgotten screw & Senior registrar & Complex fracture & Long leg cast 12 weeks \\
\hline Forgotten screw & Consultant & Anterior longitudinal fracture & Long leg cast 6 weeks \\
\hline Difficult removal & Consultant & Proximal posterior fissure & NWB 4 weeks \\
\hline Difficult removal & Senior registrar & Broken bone block & NWB 4 weeks \\
\hline Difficult removal & Senior registrar & Anteromedial fissure & NWB 8 weeks \\
\hline Undetected new bone formation in distal nail & Consultant & Oblique fracture at primary fracture site & Long leg cast 12 weeks \\
\hline
\end{tabular}

*NWB, non-weight bearing.

hardly forget. When reporting and analyzing all complications objectively, there is a possibility to learn from other surgeon's errors. As this knowledge and experience becomes more common, it will increase the quality of trauma care.

It seems that assuming the broken interlocking screw to tilt is an unnecessary risk. Brumback [28] stated that: "Removal of an interlocking nail with a portion of an interlocking screw visibly protruding from an interlocking screw hole should never be attempted." Considering the results of our study, we agree and recommend the removal of all broken screw parts prior to the extraction of the nail.

The possibility of nail rotation while attaching the extraction instrument is unlikely due to IMN's anatomic anterior bend. We suggest removing all distal locking screws at the same time instead of leaving one as anti-rotational screw for later removal and thus, increasing the risk of human error/distraction resulting in interlocking screws accidentally being left in place. In case of a difficult removal, we strongly recommend the use of fluoroscopy instead of forcing the IMN. If the fluoroscopy does not reveal forgotten or broken screws, one should look for new condensed bone around the IMN's distal interlocking screw holes. The new bone formation around an IMN is a mechanical barrier, which effectively forces the distal part of the nail to jam against the cortex and can cause a fracture. Stedtfeld et al [29] and Seebauer [30] reported similar problems. Unlike Stryker's $\mathrm{T} 2{ }^{\circledR}$ nail, which has three distal interlocking screw holes, the Expert Tibia Nail ${ }^{\mathbb{R}}$ has four holes. When less than all four holes at the distal end are not used, there is a possibility of increased ingrowth of bone fragments into the screw holes that have been left open $[30,31]$. When ingrowth at left-open screw holes is suspected we agree with Dr. Krettek's [31] 
Table 4

Checklist for removal of tibial intramedullary nails.

\section{Preoperatively}

Obtain recent x-rays with AP and lateral view

Identify the implant

Make sure that right instrumentation is available

Count the screws from X-rays and patient records

Measure the distance between screws from AP and lateral views

Look for new condensed bone around screw holes

Make sure that fluoroscopy is available

Plan the removal of possible broken screws/implants

Intraoperatively

Remove all screws and count the screws

Inspect the removed screws

Remove broken screws parts

When ingrowth in an exposed screw hole is suspected, empty the exposed hole

recommendation on emptying the nail using a long Kirschner wire and/or emptying left-open holes with a drill.

There were three failed attempts nail removal. In one case, appropriate instrumentation was not available for a nail that had been implanted in another hospital. This should have been taken in consideration in preoperative planning. In two cases the position of the nail was such that removal would have needed excessive chiseling and destruction of bone. In both cases the preoperative $\mathrm{X}$ rays showed the nail was buried deep in the tibia. In cases where the IMN is buried deeply, the surgeon should pre-operatively consider the tradeoff between the risks and benefits of the IMN's removal.

Finally, the ability of the surgeon to be able to identify possibly broken or oblique interlocking screws and bone ingrowth is of major importance to preventing complications. We must emphasize the preoperative planning. Firstly, there needs to be a protocol in place where the number of screws is counted from both AP- and lateral views and patient records. Second, attention needs to be placed on the distance between distal interlocking screws in APand lateral X-ray views, because the oblique interlocking screw can go unnoticed on plain X-ray views, especially when using only one view, as presented on Fig. 1. The presence of fibular or other hardware can make identification of all distal interlocking screws difficult especially when oblique distal interlocking screw is present. Therefore, we suggest the use of checklists (Table 4.) in preoperative planning which account for the number of interlocking screws in all $\mathrm{x}$-ray views and from patient records. Another essential element of the checklist would be the careful inspection of interlocking screws after removal in order to reveal broken screws. This would assist in avoiding fractures caused by interlocking screw parts accidently left behind.

In our study two patients who had previously been asymptomatic developed anterior knee pain at the incision site following the IMN removal. Infrapatellar nerve damage was found in both patients. There are reports of injury to the infrapatellar branch of the saphenous nerve after tibial intramedullary nailing [10]. The course of the infrapatellar nerve makes it susceptible to iatrogenic injury during extraction and insertion, when medial and midline incisions are being used [32,33]. This complication can hardly be avoided by using checklists and several authors have reported knee pain with horizontal incisions as well [32].

This study has several limitations. Its retrospective nature is the most prominent one. Due to poor documentation, we could not count the number of times when fluoroscopy was used, and for the same reason we were not able to determine the exact length of surgery. At our institute, X-rays are not routinely taken after IMN removals, so there is a possibility that some small fissures have gone unnoticed. In many cases the records just stated that it was a difficult removal but no explanation of details or reasons behind difficulties was reported. But a relatively large study population of a single tertiary care trauma unit made it possible to analyze iatrogenic fractures that had clinical significance and thus give reliable incidence of mechanical complications associated with IMN removal surgery.

\section{Conclusion}

Nail removal can be a challenging operation which does not always receive the necessary preoperative planning or operative expertise. The majority of the iatrogenic fractures in this study were avoidable. We suggest the use of checklists in preoperative planning to avoid fractures caused by broken or undetected screws.

\section{Conflicts of interest}

None. This research received no funding from Helsinki University and no grants from any funding agencies in the commercial or not-for-profit sectors.

\section{Acknowledgments}

AS: planning of the study design, data collection, statistical analysis, and preparation of manuscript. TB: planning of the study design, data collection, and preparation of manuscript LH: planning of the study design and preparation of manuscript.

\section{References}

[1] Praemer A., Furner S, Rice DP. Musculoskeletal Conditions in the United States. Amer Academy of Orthopaedic; 1999

[2] Karladani AH, Granhed H, Edshage B, Jerre R, Styf J. Displaced tibial shaft fractures: a prospective randomized study of closed intramedullary nailing versus cast treatment in 53 patients. Acta Orthop Scand 2000;71(2):160-7, doi:http://dx.doi.org/10.1080/000164700317413139.

[3] Finkemeier CG, Schmidt AH, Kyle RF, Templeman DC, Varecka TF, A prospective, randomized study of intramedullary nails inserted with and without reaming for the treatment of open and closed fractures of the tibial shaft. J Orthop Trauma 2000;14(3):187-93.

[4] Krettek C, Könemann B, Schandelmaier P, Tscherne Versorgung der $\mathrm{H}$. Unterschenkelschaftfraktur mit Unaufgebohrtem Tibianagel(UTN) und distalem zielgerät (DAD). Bilanz Zur Jahrtausendwende, 1999. Berlin, Heidelberg: Springer Berlin Heidelberg: Deutsche Gesellschaft für Chirurgie 1999. p. 1322-3, doi:http://dx.doi.org/10.1007/978-3-642-60248-1_349.

[5] Oedekoven G, Claudi B, Frigg R. Die Osteosynthese der instabilen offenen und geschlossenen Tibiafraktur mit ungebohrtem Tibiaverriegelungsnagel. Operat Orthopädie und Traumatologie 1992;4(2):86-99, doi:http://dx.doi.org/ 10.1007/BF02513414.

[6] Böstman O, Pihlajamäki H. Routine implant removal after fracture surgery: a potentially reducible consumer of hospital resources in trauma units. JTrauma 1996:41(5):846-9.

[7] Boerger TO, Patel G, Murphy JP. Is routine removal of intramedullary nails justified. Injury 1999;30(2):79-81.

[8] Court-Brown CM, Gustilo T, Shaw AD. Knee pain after intramedullary tibial nailing: its incidence, etiology, and outcome. J Orthop Trauma 1997;11 (2):103-5, doi:http://dx.doi.org/10.1097/00005131-199702000-00006.

[9] Folwaczny $\mathrm{EF}$, Stürmer KM. Unusual complications during removal of intramedullary nails after lower leg fractures tibial shaft fissure and inability to remove the nail. Eur J Trauma 2001;27(4):184-90, doi:http://dx doi.org/10.1007/s00068-001-1058-y.

[10] Karladani AH, Ericsson PA, Granhed $H$, Karlsson L, Nyberg P. Tibial intramedullary nails - should they be removed? A retrospective study of 71 patients. Acta Orthop 2007;78(5):668-71, doi:http://dx.doi.org/10.1080/ 17453670710014374.

[11] Sidky A, Buckley RE. Hardware removal after tibial fracture has healed. Can J Surg 2008;51(4):263-8

[12] Hora K, Vorderwinkler KP, Vécsei V, Gäbler C. Intramedullary nail removal in the upper and lower limbs. Should we recommend this operation? Unfallchirurg 2008;111(8), doi:http://dx.doi.org/10.1007/s00113-008-1450-8 599-601-603-5.

[13] White A, Bishay M, Jones CB. Complication of removal of tibial nails. Injury 1998;29(2):165-6, doi:http://dx.doi.org/10.1016/S0020-1383(98)00045-X.

[14] Zenios M, Malik MHA, Al-Mesri AR, Vhadra R, Khan SA. Current intramedullary nail insertion and removal practice in the UK. Eur J Orthop Surg Traumatol 2004;14(1):19-22, doi:http://dx.doi.org/10.1007/s00590-003-0122-1.

[15] Vos DI, Verhofstad MH, Hanson B, van der Graaf Y, van der Werken C. Clinica outcome of implant removal after fracture healing. Design of a prospective multicentre clinical cohort study. BMC Musculoskelet Disord 2008 9:1 2012;13 (1):147, doi:http://dx.doi.org/10.1186/1471-2474-13-147. 
[16] Hanson B, van der Werken C, Stengel D. Surgeons' beliefs and perceptions about removal of orthopaedic implants. BMC Musculoskelet Disord 2008 9:1 2008;9(1):73, doi:http://dx.doi.org/10.1186/1471-2474-9-73.

[17] Vos DI. Implant removal after fracture healing: facts and fiction. Utrecht University Repository; 2013.

[18] Onche II, Osagie OE, I.Nuhu S. Removal of orthopaedic implants: indications outcome and economic implications. J West Afr Coll Surg 2011;1(1):101-12.

[19] Pathak SK, Maheshwari PMP, Gour S, Gadi D. Intramedullary nails: should it be removed after fracture healing? Int Surg J 2016;3(3):1603-5, doi:http://dx.doi. org/10.18203/2349-2902.isj20162755.

[20] Richards RH, Palmer JD, Clarke NMP. Observations on removal of metal implants. Injury 1992;23(1):25-8, doi:http://dx.doi.org/10.1016/0020-1383 (92) $90120-\mathrm{H}$

[21] Jones DH, Schmeling G. Tibial fracture during removal of a tibial intramedullary nail. J Orthop Trauma 1999;13(4):271-3.

[22] Brown RM, Wheelwright EF, Chalmers J. Removal of metal implants after fracture surgery-indications and complications. J R Coll Surg Edinb 1993;38 (2):96-100.

[23] Sohlberg R, Abraham E. Tibia fracture caused by a broken screw during interlocking intramedullary nail extraction. Orthopedics 1995;18(6):575-6, doi:http://dx.doi.org/10.3928/0147-7447-19950601-13.

[24] Takakuwa M, Funakoshi M, Ishizaki K, Aono T, Hamaguchi H. Fracture on removal of the ACE tibial nail. J Bone Jt Sur - Br Vol 1997;79(3):444-5.
[25] Im G-I, Lee K-B. Difficulties in removing ACE tibial intramedullary nail. Int Orthop 2003;27(6):355-8, doi:http://dx.doi.org/10.1007/s00264-003-0503-4.

[26] Sourlas L, Papadakis M, Lallos S, Brilakis E. Efstathopoulos N: tibial shaft fracture after removal of an ACE tibial nail.J Long Term EffMed Implants. 2011;21(3):193-6.

[27] Sanderson PL, Ryan W, Turner PG. Complications of metalwork removal. Injury 1992;23(1):29-30.

[28] Brumback RJ. Removal of broken interlocking screws. Tech Orthopaedics 2001;16(4):349.

[29] Stedtfeld HW, Landgraf P. The impacted intramedullary nail. The detection of an old problem in a new nail. Unfallchirurg 1998;101(12):950-4, doi:http://dx. doi.org/10.1007/s001130050362.

[30] Seebauer CJ, van Scherpenzeel KM, Haas NP, Bail HJ. Tibia fracture following removal of the ETN ${ }^{\mathbb{R}}$ (Expert Tibia Nail): a case report. Arch Orthop Trauma Surg 2009;129(7):949-53, doi:http://dx.doi.org/10.1007/s00402-008-0805-5.

[31] Krettek C, Mommsen P. Implant removal after intramedullary osteosyntheses. Literature review, technical details, and tips and tricks. Unfallchirurg 2012;115 (4):299-314, doi:http://dx.doi.org/10.1007/s00113-012-2158-3.

[32] Leliveld MS, Verhofstad MHJ. Injury to the infrapatellar branch of the saphenous nerve, a possible cause for anterior knee pain after tibial nailing? Injury 2012;43(6):779-83, doi:http://dx.doi.org/10.1016/j.injury.2011.09.002.

[33] Dunaway D, Steensen R, Wiand W, Dopirak R. The sartorial branch of the saphenous nerve: its anatomy at the joint line of the knee. Arthroscopy 2005;21(5):547-51, doi:http://dx.doi.org/10.1016/j.arthro.2005.02.019. 